\title{
e-Phaïstos
}

e-Phaïstos

Revue d'histoire des techniques / Journal of the history

of technology

VII-2 | 2019

La sauvegarde du patrimoine scientifique

\section{Pour une socio-anthropologie des techniques}

For a Socio-Anthropology of Technics

\section{Caroline Moricot}

\section{OpenEdition}

Journals

Édition électronique

URL : http://journals.openedition.org/ephaistos/5133

DOI : 10.4000/ephaistos.5133

ISSN : 2552-0741

Éditeur

IHMC - Institut d'histoire moderne et contemporaine (UMR 8066)

Référence électronique

Caroline Moricot, « Pour une socio-anthropologie des techniques », e-Phaïstos [En ligne], VII-2 | 2019,

mis en ligne le 15 octobre 2019, consulté le 09 novembre 2019. URL : http://journals.openedition.org/ ephaistos/5133

Ce document a été généré automatiquement le 9 novembre 2019.

Tous droits réservés 


\title{
Pour une socio-anthropologie des techniques
}

For a Socio-Anthropology of Technics

\author{
Caroline Moricot
}

\author{
HDR : références bibliographiques \\ Caroline MORICOT, Pour une socio-anthropologie des techniques, Habilitation à \\ diriger des recherches en sociologie, Université Paris 1 Panthéon Sorbonne, \\ soutenue le 14 mai 2018, 3 volumes (628 p).

\section{Garant :} \\ Thierry PILLON, Université Paris 1 Panthéon Sorbonne \\ Jury : \\ Céline LAFONTAINE, Université de Montréal, \\ Anne MONJARET, CNRS, IIAC, \\ François VATIN, Université Paris Ouest Nanterre, \\ Pascal VENESSON, RSIS, Nayang Technological University et Université Paris 2 \\ Panthéon Assas), \\ Dominique VINCK, Université de Lausanne
}

1 La présentation d'un dossier d'habilitation à diriger des recherches invite à un retour sur son propre parcours, construit par les rencontres qui le façonnent et l'orientent. "Les sociétés se ressentent toujours des conditions qui les ont vues naître ", écrivait Tocqueville. Je pense qu'il en va de même pour les individus et, pour ma part, je suis née à la sociologie dans les années 1980 à Paris V. Je réalise combien ces années de formation ont été déterminantes de ma façon de penser jusqu'à aujourd'hui.

2 En tant que sociologue, je m'intéresse à la société de la modernité, celle que Georges Balandier caractérisait comme traversée par, à la fois, le mouvement et l'incertitude. Son invitation à en explorer les "nouveaux nouveaux mondes " avec le regard d'un anthropologue qui découvrirait une nouvelle civilisation, celle que pourtant il habite, 
mais qui lui est, par bien des aspects, étrangère, constitue pour moi un fil directeur, voire un moteur.

Dans cette perspective, l'un des objets les plus fascinants et les plus complexes de notre société est le rapport qu'elle entretient avec la technique. Cette dernière opère comme « un fait social total», selon la formule de Marcel Mauss, traversant tous les domaines du social, économique comme juridique, recomposant les sphères du public et du privé, influençant les relations au sein de la famille comme de l'entreprise, interrogeant les modalités d'accès au savoir et de production des connaissances, bousculant le rapport au temps et à l'espace. Elle participe de la déconstruction comme de la reconstruction du lien social, introduisant de nouveaux objets dans notre quotidien, ébranlant les hiérarchies en place et bouleversant le statut du proche et du lointain. L'imaginaire qui nourrit les mondes techniciens est aujourd'hui lié aux notions de progrès, d'efficacité, de puissance, mais aussi de contrôle, de contrainte des savoir-faire, de dépendance. Cette tension est inscrite dans les usages des objets techniques, mais aussi dans les processus de leur conception qui participent de leur destinée. Les différents objets sur lesquels j'ai eu à travailler portent tous cette tension incarnée dans le réseau des acteurs qui les conçoivent, les utilisent, les réglementent et, plus largement, les inscrivent dans un système de significations souvent non consensuel. Ils ont offert à mon regard leur potentiel d'arbitraire et d'indéterminé, leur caractère fondamentalement social, celui qu'il revient au sociologue de faire resurgir inlassablement et dont la naturalité des pratiques est à déconstruire.

4 Si je devais résumer en quelques mots mon travail de recherche, je dirais qu'il est motivé par une triple préoccupation: celle de l'objet, celle de l'approche et celle de l'enjeu.

5 Mes objets de recherche sont des objets techniques, des avions, des drones, des robots chirurgicaux, mais aussi des écrans, des interfaces, des images... Ils constituent toujours pour moi le point d'entrée.

6 Mon approche est celle d'une sociologue de terrain qui observe et qui écoute pour tenter de comprendre le point de vue des acteurs sur leur activité (c'est-à-dire sur leur pratique, leurs représentations, leur imaginaire...). De ce point de vue, ma contribution à une sociologie des techniques s'ancre toujours à partir d'un point de vue local, celui de l'expérience des acteurs que j'ai patiemment recueillie dans la durée. Je suis d'abord une sociologue de terrain au sens où c'est toujours à partir de l'engagement sur un terrain que se construit ma réflexion, que mes questions de départ se problématisent. Un engagement sur le terrain qui est souvent collectif, c'est-à-dire qui se fait au sein d'une équipe, parfois pluridisciplinaire, et je remercie ceux qui ont partagé avec moi ce compagnonnage de recherche dont je retiens d'abord la richesse et la stimulation.

7 Les enjeux soulevés par mes recherches sont centralement celles des techniques automatisées dans ce qu'elles font aux opérateurs qui, dans leur activité quotidienne et ordinaire, y sont confrontés dans un processus d'appropriation. Comment en particulier ces techniques s'adressent-elles à leur corps? Au sein des processus automatisés, une partie de l'activité s'accomplit indépendamment de l'action des opérateurs, c'est-à-dire de façon autonome. On pourrait penser alors que les automates se substituent aux humains. Pourtant, c'est le contraire que j'ai observé : parmi les résultats de ma recherche sur le pilotage des avions de nouvelle génération dans l'aviation civile, il est un constat qui peut paraitre paradoxal ou du moins inattendu : cette intensification des automatismes dans les glass-cockpits rend la présence des 
pilotes encore plus nécessaire. De plus, les automatismes rendent cette présence exigeante et pourtant, à bien des égards, ils ne la facilitent pas. C'est le même constat que j'ai fait récemment à propos des usages des drones dans l'armée de l'air française. Le projet d'automatisation porte en lui un idéal de perfection : l'automate serait un système sûr, constant, infaillible... Mais cette définition de la perfection ne peut-elle être remise en cause ? Est-elle suffisante ? À la suite du philosophe des techniques Gilbert Simondon, j'avais tenté de montrer que le véritable perfectionnement des systèmes techniques complexes réside, non pas dans l'augmentation du nombre ou de l'importance de leurs automatismes, mais dans le fait qu'ils recèlent une certaine "marge d'indétermination" et que, grâce à cette marge d'indétermination, ils maintiennent une "sensibilité au monde ». Il revient aux opérateurs, dit Simondon, de s'immiscer dans cette marge d'indétermination. Autrement dit, c'est parce que les machines sont «incomplètes" qu'elles portent un potentiel de perfection; et ce perfectionnement, elles l'acquièrent dans ce que les hommes font d'elles. Ce sont les gestes que les opérateurs effectuent autour et avec les machines qui leur donnent un sens. C'est à la lumière de cette conception inhabituelle de l'automatisation que j'ai construit ma réflexion.

8 Car à côté de l'horizon d'une automatisation globale régulièrement réactivé comme une promesse par les imaginaires techniciens et en particulier dans les moments d'innovation, un autre constat se donne à voir. Si l'on regarde de près les situations socio-techniques les plus communes et les plus courantes, on se rend très vite compte que l'on a affaire à des situations mixtes où les automates se substituent en effet pour une part à l'activité des hommes, mais ne savent pas se priver de leur présence, moins pour fonctionner, mais pour que leur fonctionnement ait un sens. Dans un avion, le pilote surveille les systèmes, les contextualise; il récupère nombre de situations déviantes ; bref, il adapte et ajuste en temps réel un plan de vol, c'est-à-dire un scénario «idéal» qui se déroule sur les écrans du cockpit. Il est important de continuer à travailler sur ces situations (que certains qualifieront peut-être de situations de transition), de continuer à les penser: elles sont les plus nombreuses, elles sont durables, elles caractérisent, au-delà des milieux aéronautiques, un grand nombre de situations de travail. Il faut donc continuer à penser ces situations, les plus communes, et à penser particulièrement la place, c'est-à-dire le mode de présence des humains dans ces dispositifs (et en particulier la place accordée à leur corps). Pour caractériser cette présence, on pourrait dire que l'opérateur se trouve au cœur d'une tension, qu'il lui revient finalement de gérer, entre une tendance de l'automatisation qui vise à l'écarter et une gestion quotidienne des systèmes qui, dans le même temps, exige sa présence et la rend irréductible. Ce paradoxe de l'automatisation participe de la définition des humains dans ces grands systèmes techniques que j'ai étudiés; des systèmes où le risque est omniprésent et où la sécurité est un enjeu impératif. Ce ne sont pourtant pas les accidents et la construction des causalités qui les ont fabriqués que j'ai analysé pour envisager la question de la sécurité. Je suis partie de l'autre bout de la chaîne pour aborder la sécurité sous l'angle de sa construction quotidienne par l'efficacité conjuguée de collectifs de travail, plutôt que sous l'aspect exceptionnel des accidents ou incidents qui montrent, eux, comment la sécurité est déconstruite, défaite. Il est important d'expliquer les moments de ruptures, les enchaînements fatals pour essayer de les prévenir dans le futur. Il est tout aussi important d'expliquer pourquoi et comment, en règle générale, la continuité est assurée. Ces deux types d'analyses qui 
partent aux antipodes l'une de l'autre devraient pouvoir se rejoindre au lieu d'être rivales.

9 Ma réflexion ne s'est jamais attachée à une seule école de pensée ; elle s'autorise à " faire feu de tout bois » (selon le terme de Jean-Pierre Olivier de Sardan) et vise à tisser des liens entre différentes approches sociologiques et anthropologiques, mais aussi philosophiques et historiques. Ainsi, je retiens, entre autres, la sociologie de l'usage de Victor Scardigli pour son ancrage dans le quotidien, la philosophie des techniques de Gilbert Simondon pour la formalisation de la question des marges d'indétermination des objets techniques, l'anthropologie d'André-Georges Haudricourt pour son insistance sur les gestes qui entourent les objets et leur donnent un sens, la sociologie de la traduction de Madeleine Akrich pour l'engagement à penser ensemble la conception et l'usage, la sociologie pragmatique de Nicolas Dodier pour les concepts de solidarité technique et d'arène des habiletés, la socio-anthropologie de Pierre Bouvier pour l'invitation au décentrement et au dépassement des frontières qu'elle porte, la sociologie des macro système techniques d'Alain Gras pour son ouverture sur les relations de dépendance dans les réseaux, l'anthropologie du corps de Mauss pour son intuition fondamentale que le corps est dans les techniques, la sociologie dynamique pour l'attention aux mouvements qui traversent le social, aux tensions qu'il ne faut pas masquer mais qu'il faut travailler. Vous pourriez penser que j'omets la sociologie du travail. Il est vrai que mes rencontres n'ont pas été d'abord celle-là. Certes, mes objets sont liés aux mondes professionnels (celui des pilotes de ligne, des pilotes de chasse, des opérateurs de drones, des chirurgiens, des anatomo-pathologistes), mais c'est par le prisme de la technique que j'y suis d'abord entrée et ce sont prioritairement les enjeux liés aux effets du changement technique sur le corps des opérateurs que j'ai exploré. Ces questions, certes présentes dans certains courants de la sociologie du travail, n'étaient pas, à mon sens, suffisamment traitées en termes de représentations sociales, d'imaginaire mobilisé, d'engagement du corps. Un rapprochement a tout son sens aujourd'hui avec le courant de la sociologie de l'activité issu d'une sociologie du travail, plus attentive aux circonstances du travail en actes et aux enjeux techniques et corporels qu'il mobilise.

10 La question de l'action à distance est donc au cœur du travail que je présente dans le mémoire original et je voudrais en venir maintenant à l'exposé des enjeux que cette question soulève d'un point de vue socio-anthropologique en commençant par rappeler que mon travail s'appuie sur deux enquêtes de terrain qui n'avaient pas initialement vocation à être associées et dont j'ai revisité les données pour asseoir ma réflexion. La première étudie deux cas: la robotique chirurgicale et la fabrication des biopsies optiques pour les anatomo-pathologistes et la seconde porte sur les drones militaires. C'est a posteriori que l'exercice de confrontation des données a été réalisé à partir de l'hypothèse que ces différents acteurs, chacun dans leur milieu singulier et forgés par une histoire collective originale des relations à la technique, font l'expérience de la pratique de l'action à distance. L'enjeu commun à ces terrains est que la combinaison d'objets techniques de plus en plus performants, alliés à la puissance des calculateurs (qui modélisent les processus et les rendent ainsi en théorie universels et transposables) et à la transmission instantanée de données ont rendu possible le fait d'être ici et de projeter son action ailleurs. Quels sont les effets de cette transformation de la définition de l'action désormais étendue à toutes les formes de présence à distance? Je m'intéresse ici à un aspect particulier de cette transformation: le découplage du geste et de la matière agit en questionnant les conséquences sur 
l'organisation des collectifs de travail, leurs représentations de l'activité et la nature du lien social fabriqué par cette situation.

11 Certains ont espéré que les machines remplaceraient un jour les opérateurs et d'autres l'ont craint. D'un côté, étaient mise en avant l'inconstance et l'indiscipline de la nature humaine et, de l'autre, son ingéniosité et sa capacité d'adaptation. Cette tension entre deux visions, faire ou faire-faire, a traversé tout le $\mathrm{XX}^{\mathrm{e}}$ siècle, polarisant le débat politique, économique, mais aussi social et même sociologique. Il a également nourri les promesses et les craintes relatives aux projets d'action à distance initiés dans les années 1960, et ce débat se rejoue aujourd'hui au sein même de la communauté des concepteurs de ces systèmes, entre « roboticiens » et " coboticiens ».

C'est comme souvent dans l'entre-deux de cette tension qu'il faut explorer, pour mieux comprendre les choses et en prendre la mesure. Car, encore une fois, force est de constater, que, d'une part, les machines, si elles prennent effectivement en charge une partie de l'action de manière autonome, ne fonctionnent pas toutes seules et que, d'autre part, les opérateurs sont toujours là, et parfois même plus nombreux comme dans le cas des drones. Ce que révèle et cristallise cette tension, c'est la place (et en conséquence la visibilité) que l'on souhaite et que l'on décide d'accorder au corps des opérateurs dans la conduite de l'activité. Entre exposition et protection, entre effort et ennui, entre guidage et surveillance, toute une palette nuancée d'engagement dans l'action est possible.

Dans les procédures d'action à distance, les opérateurs sont certes présents, mais derrière des écrans et des postes de commande. Ce que modifie profondément leur mise à distance, c'est la spatialité du travail ou, autrement dit, l'organisation spatiale de l'activité, entre zone sûre et confortable et zone hostile ou inaccessible et donc interdite. La zone sûre est, dans une logique de protection, celle où se trouve l'opérateur qui agit sur les interfaces de commande, et la zone interdite est celle où se trouve la machine qui reçoit les ordres - les interprète pour les optimiser en annulant par exemple le tremblement naturel de la main du chirurgien - et agit sur la matière. Cette spatialité des systèmes d'action à distance s'adresse donc directement au corps de ceux qui les utilisent. La division de l'espace en deux zones distinctes et hermétiquement séparées relève d'une construction technique, mais aussi sociale de l'activité qui n'est pas sans faire écho aux remarques de l'anthropologue Mary Douglas sur la fabrication sociale du propre et du sale, de la souillure et du tabou. L'action à distance prend, en effet, le parti de protéger les corps des opérateurs (non plus en les équipant, mais en les excluant), mais cela a un prix, celui de leur confinement, d'une part, et celui, d'autre part, d'une division de l'activité entre, pour le dire vite, l'environnement à portée de leur main (celui des interfaces) et l'environnement inaccessible de la machine agissante.

Le maintien des liaisons entre les deux espaces, associés par la temporalité de l'activité, prend alors une place essentielle, tant dans le fonctionnement technique des systèmes qui doivent être reliés de façon stable et continue, que dans les coordinations entre les opérateurs disséminés à différents endroits du réseau. Ainsi, les images captées par le drone et instantanément diffusées à la hiérarchie militaire, sont enrichies et accompagnées de messages visant à les replacer dans un contexte et à s'assurer qu'elles ne feront pas l'objet d'interprétation trop rapide ou erronée par le commandement. Quant au chirurgien aux commandes du robot, il n'a plus de contact visuel avec son équipe qui se tient près du lit du patient (ses yeux sont plongés dans le corps du patient 
grâce aux lunettes de visualisation 3D) et c'est exclusivement à la voix qu'il doit communiquer, formuler ses attentes et maintenir son autorité.

C'est cette question du maintien de l'activité dans un monde commun que soulèvent les opérateurs de drone français lorsqu'ils insistent sur la « colocalisation » de l'équipage qui, s'il ne peut plus être embarqué à bord du drone, peut au moins opérer depuis le théâtre d'opération, c'est-à-dire depuis une zone en guerre. Le même type d'organisation se remarque dans l'usage en routine du robot chirurgical dont la console de commande se trouve dans la salle d'opération divisée en conséquence en deux parties, stérile ou non, le chirurgien se trouvant dans cette dernière. Certes, la démonstration d'un usage à grande distance a été faite il y a déjà plus de quinze ans, mais ce n'est pas l'usage courant et cela ne tient pas seulement à son approbation réglementaire, mais au moins tout autant à l'importance, aux yeux des opérateurs, de maintenir non seulement une temporalité, mais aussi l'horizon d'une spatialité commune avec la finalité de leur action. Il s'agit, en un mot, d'être présent aux machines sans perdre le fait d'être présent à la mission. Cela semble particulièrement essentiel dans ces métiers où la vie et la mort sont en jeu. Pourtant, la reprise en main n'est déjà plus possible en cas de problème (la mission du drone est abandonnée; le chirurgien à la console ne pourra approcher immédiatement le patient pour convertir l'opération) et l'on pourrait considérer que la véritable rupture s'est déjà produite. La volonté de maintenir cette proximité, même si elle n'en est déjà plus vraiment une, souligne l'importance du lien, d'une solidarité (avec l'équipe et avec le patient ; avec les troupes au sol, voire avec l'ennemi) qui menace d'être fragilisée (les Américains opèrent déjà leurs drones depuis leur territoire national ; la réglementation sur un usage du robot chirurgical à grande distance pourrait être finalement obtenue) et l'insistance des opérateurs démontre une évolution possible et redoutée. Dans le cas des drones, l'argument économique (ce sera moins cher) rejoint l'argument technique (on sait le faire) auquel s'oppose encore l'argument politique et éthique (on ne fait pas la guerre depuis son bureau). Dans le cas de la chirurgie, le coût d'achat, mais aussi d'utilisation du robot est tellement élevé que l'argument économique ne peut plaider en sa faveur, en tout cas dans l'organisation actuelle qui ne laisse pas entrevoir, comme dans le cas des drones militaires, un basculement potentiel à court terme de l'organisation du travail.

Les chirurgiens aujourd'hui formés à la pratique robotique ont l'expérience de la chirurgie laparoscopique et/ou de la chirurgie ouverte; les pathologistes ont l'expérience de la lecture des lames qu'ils ont fabriqué à partir de la pièce opératoire ; les pilotes des drones sont des pilotes de chasse reconvertis et leur expérience passée lors de missions opérationnelles leur est très utile dans le cadre de la pratique du drone. C'est cette expérience passée qui leur permet d'inscrire leur pratique à distance dans une dynamique qu'ils connaissent finement et jusque dans les sensations corporelles du vol ou de la résistance des tissus sous la lame du scalpel, qu'ils peuvent alors imaginer ressentir mais qu'ils ne ressentent plus.

17 La vue est depuis toujours l'organe du contrôle à distance. Elle est, avec ces dispositifs d'action, amplifiée (zoom de la caméra du drone, image numérique à l'échelle cellulaire sur l'écran du pathologiste) et magnifiée (image 3D du robot chirurgical) dans le but de donner à l'opérateur la sensation d'être immergé là où il n'est pas, une immersion visuelle qui vise à effacer la distance par ailleurs soigneusement construite. Si la vue est sollicitée à tout instant dans la pratique à distance, c'est parce qu'il ne faut pas lâcher 
du regard le fil de l'action, que l'on ne peut plus percevoir autrement, et en particulier par le toucher (dans le cas de la chirurgie robotique ou du pathologiste analysant des biopsies optiques) ou par le ressenti des effets du vol (le facteur de charge par exemple chez les pilotes de drone). La main de l'opérateur n'est pas pour autant complètement désinvestie; elle agit dans le cadre de son périmètre corporel sur les interfaces de commande qui vont relayer son geste. Il n'est plus nécessaire de déployer de la force ou de l'amplitude et, en contrepartie, le geste co-opéré avec la machine et assisté par un retour visuel de qualité, gagne en précision et en finesse. On peut lire dans cette observation de la posture de l'opérateur à distance la contrainte d'un double mouvement consistant à la fois en un rétrécissement de son geste et dans une projection des effets de celui-ci au-delà des limites de son environnement immédiat. Cette mise en tension procède d'un engagement dans l'action qui fait appel à de nouvelles compétences mobilisant à la fois les corps et les techniques dans des formes d'association nouvelles et pour une grande part encore à continuer de définir et d'observer dans leur usage.

\section{RÉSUMÉS}

La combinaison d'objets techniques de plus en plus performants, alliés à la puissance des calculateurs (qui modélisent les processus et les rendent ainsi en théorie universels et transposables) et à la transmission instantanée de données ont rendu possible le fait d'être ici et d'agir/de projeter son action ailleurs. Quels sont les effets de cette transformation de la définition de l'action désormais étendue à toutes les formes de présence à distance? Je m'intéresse ici à un aspect particulier: le découplage du geste et de la matière agie en questionnant les conséquences sur l'organisation des collectifs de travail, leurs représentations de l'activité et la nature du lien social fabriqué par cette situation. Dans les processus automatisés, une partie de l'action se déroule de façon autonome, c'est-à-dire sans intervention humaine directe. D'autres moments supposent une présence active de l'opérateur humain. Se posent alors la question de la vigilance, celle de la reprise en main, mais aussi celle de la responsabilité et de l'engagement. Les modes automatisés ont initié le fait que le corps soit tenu à distance de l'action tandis que les modes manuels le convoquaient sans relâche. C'est cette même mise à distance qui se prolonge et se radicalise avec la télé-chirurgie ou avec les usages militaires des drones, deux terrains à partir desquels s'est construite mon analyse. Jusqu'à quel point le corps à corps avec la matière est-il nécessaire à la prise en compte de l'altérité et à l'engagement de soi dans une action sur autrui dont la vie est en jeu? Et lorsqu'il n'est plus possible, quelles formes de substituts ou de traductions se mettent alors en place?

The combination of increasingly powerful technical objects combined with the power of calculators (which model processes and thus make them universal and transposable) and instant data transmission made it possible to be here and to act elsewhere. What are the effects of this transformation of the definition of action now extended to all forms of remote presence? I am interested here in a particular aspect of this transformation: the decoupling of the gesture and the acted matter by questioning the consequences on the organization of the collective work, their representations of the activity and the nature of the social link made by this situation. In 
automated processes, part of the action takes place autonomously, that is, without direct human intervention. Other moments assume an active presence of the human operator. This raises the question of vigilance, that of recovery, but also that of responsibility and commitment. The automated modes initiated the fact that the body was kept away from the action while the manual modes summoned it relentlessly. It is this same distancing that is prolonged and radicalized with telesurgery or the military uses of drones, two fields from which my analysis was built. To what extent is body-to-body contact with matter necessary for the consideration of otherness and self-commitment in an action on others whose lives are at stake? And when it is no longer possible, what forms of substitutes or translations are then put in place?

\section{INDEX}

Mots-clés : histoire des techniques, drone, télé-chirurgie, automatisation, geste technique, corps

Keywords : history of technology, drone, tele-surgery, automation, technical gesture, body Thèmes : Positions de thèse/HDR

\section{AUTEUR}

\section{CAROLINE MORICOT}

Caroline Moricot est Maîtresse de Conférences-Habilitée à Diriger des Recherches en sociologie à l'Université Paris 1 Panthéon-Sorbonne et membre du CETCOPRA (Centre d'Etude des Techniques, de COnaissances et des PRAtiques). Elle a travaillé sur les enjeux de l'appropriation des dispositifs automatisés en s'appuyant sur des terrains de recherche dans l'aéronautique civile et militaire. Elle réfléchit aujourd'hui à partir des terrains de la chirurgie robotique et des drones militaires à la question de la présence corporelle dans l'action à distance. Elle a fondé le Groupe de Travail «Corps, techniques et société » de l'Association Française de Sociologie et dirige la revue Socio-Anthropologie (Éditions de la Sorbonne). Elle a récemment publié aux PUF, avec Gérard Dubey, Dans la peau d'un pilote de chasse. Le spleen de l'homme machine (2016). 